\title{
Antioxidants and sugars - two key areas under debate
}

A ntioxidants are often presumed to be the principles behind protective effects of high fruit and vegetable intake. Intervention studies with increased fruit and vegetable intake, however, have so far failed to show clear evidence of effects on markers of oxidative stress in vivo. Antioxidants are suggested to interact in a complex network, and methods for measuring total antioxidative capacity have therefore been suggested as useful. In this issue Cecilia Nälsén and co-workers report correlations between intakes of ascorbic acid, tocopherols and $\beta$-carotene, and plasma total antioxidative capacity but no effect on lipid peroxidation in vivo, measured as levels of $\mathrm{F}_{2}$ isoprostanes, indicating that this is not easily affected by the diet. Inga Thorsdottir and coworkers showed that serum concentrations of vitamin $C$ and $\beta$-carotene in 6-year old children correlated with those of their parents, even stronger than the intake of fruit and vegetables, supporting the usefulness of these substances as markers of fruit and vegetable intake. The authors conclude that interventions aiming at increasing fruit and vegetable consumption should focus on the whole family.

Nordic nutrition recommendations have since more than 30 years set an upper limit of added refined sugars corresponding to 10 percent of the energy intake $(\mathrm{E} \%)$. The main argument is to ensure a high nutrient density providing enough essential nutrients and dietary fibre, which is especially important in children and adults with low energy intake (less than $8 \mathrm{MJ} /$ day). The other main rationale is to diminish the risk of dental caries, related especially to frequent intake of sugary products. More recently, the presumed role of sugars in drinks for excess energy intake and obesity has been an additional argument for limiting the sugar intake from such products, as expressed in the WHO TRS 916 report (2003) and the new Nordic Nutrition Recommendations 2004. Although useful for planning diets for heterogenous groups, the 10 $\mathrm{E} \%$ added sugars recommendation should be used with flexibility in evaluating diets: At moderate or high physical activity and normal body weight a higher amount may be compatible with an otherwise nutritious diet, whereas in other cases it may be difficult to accommodate even $10 \mathrm{E} \%$ added sugars.

With the present strong focus on limitations of sugar intake, the interest in sweeteners and products with sweeteners has increased. With this background, the Swedish Nutrition Foundation carried out a project on sugars and sweeteners focusing on their use in practice. Two outputs of this project are included in the present issue of the SJFN: A review entitled "Effects of dietary fructose on lipid metabolism, body weight and glucose tolerance in humans" by Tommi Vasankari and Tuula Vasankari, and a report from an expert meeting on the use in practice of sugars and sweeteners, especially in relation to obesity, diabetes and dental caries, authored by Paula Novicka and Susanne Bryngelsson. These reports will be followed by a review in a coming issue on the approved sweeteners, with a focus on the safety evaluation. We hope that these reports will contribute to a balanced debate, and to more elaborated recommendations on the use of sugars and sweeteners in practice.

Nils-Georg Asp

Editor-in-Chief

E-mail: asp@snf.ideon.se 